\title{
Statistical estimation of spatial wave extremes for tropical cyclones from small data samples: validation of the STM-E approach using long-term synthetic cyclone data for the Caribbean Sea
}

\author{
Ryota Wada ${ }^{1}$, Jeremy Rohmer ${ }^{2}$, Yann Krien ${ }^{3}$, and Philip Jonathan ${ }^{4,5}$ \\ ${ }^{1}$ The University of Tokyo, Tokyo, Japan \\ ${ }^{2}$ BRGM, Orleans, France \\ ${ }^{3}$ SHOM, DOPS/HOM/REC, Toulouse, France \\ ${ }^{4}$ Shell Research Limited, London SE1 7NA, United Kingdom. \\ ${ }^{5}$ Department of Mathematics and Statistics, Lancaster University LA1 4YF, United Kingdom. \\ Correspondence: Ryota Wada (r_wada@k.u-tokyo.ac.jp)
}

\begin{abstract}
Occurrences of tropical cyclones at a location are rare, and for many locations, only short periods of observations or hindcasts are available. Hence, estimation of return values (corresponding to a period considerably longer than that for which data is available) for cyclone-induced significant wave height (SWH) from small samples is challenging. The STM-E (space-time maximum and exposure) model was developed to provide reduced bias in estimates of return values compared to

5 competitor approaches in such situations, and realistic estimates of return value uncertainty. STM-E exploits data from a spatial neighbourhood satisfying certain conditions, rather than data from a single location, for return value estimation.

This article provides critical assessment of the STM-E model for tropical cyclones in the Caribbean Sea near Guadeloupe for which a large database of synthetic cyclones is available, corresponding to more than 3,000 years of observation. Results indicate that STM-E yields values for the 500-year return value of SWH and its variability, estimated from 200 years of cyclone data, consistent with direct empirical estimates obtained by sampling 500 years of data from the full synthetic cyclone database. In general, STM-E also provides reduced bias and more realistic uncertainty estimates for return values relative to single location analysis.
\end{abstract}

KEYWORDS: tropical cyclone spatial extremes synthetic storm return value

\section{Introduction}

Tropical cyclones (also named hurricanes or typhoons depending on the region of interest) are one of the deadliest and most devastating natural hazards that can significantly impact lives, economies and the environment in coastal areas. In 2005, hurricane Katrina, which hit New Orleans, was the most costly natural disaster of all time for the insurance sector, with losses totalling more than $10^{11}$ US dollars (Barbier 2015). In 2017, hurricanes Harvey, Irma and Maria caused record losses within just four weeks totalling more than $9 \times 10^{10} \mathrm{US}_{\text {dollars }}{ }^{1}$. Tropical cyclones present multiple hazards, including large damaging

\footnotetext{
${ }^{1}$ https://www.munichre.com/en/risks/natural-disasters-losses-are-trending-upwards/hurricanes-typhoons-cyclones.html-1979426458
} 
winds, high waves, storm surges, and heavy rainfall, as exemplified by Typhoon Hagibis in Japan (see context description in Dasgupta et al. 2020) or Cyclone Idai in Mozambique in 2019. ${ }^{2}$

Waves are one of the major hazard associated with tropical cyclones, of critical importance regarding marine flooding, especially for volcanic islands like those in the Lesser Antilles - North Atlantic ocean basin (Krien et al. 2015), in the Hawaï - Northeast Pacific ocean basin (Kennedy et al. 2012), or in the Reunion Island - Southwest Indian ocean basin (Lecacheux et al. 2021). Here, the absence of a continental shelf and the steep coastal slopes limit the generation of high atmospheric storm surge, but increase the potential impact of incoming waves. Moreover, wind-waves propagate with little loss of energy over the deep ocean: this might potentially increase the spatial extent as well as time duration over which damaging coastal impacts occur during a tropical cyclone event (Merrifield et al. 2014); this contrasts with tropical cyclone-induced storm surge, which tends to be concentrated in the vicinity of the cyclone centre.

To help decision makers in diverse fields such as wastewater management, transport and infrastructure, health, coastal zone management and insurance, one key ingredient is the availability of data for the frequencies and magnitudes of extreme cyclone-induced coastal significant wave heights SWH, e.g. the 100-year return levels (see an illustration in Reunion Island by Lecacheux et al. 2012: Figure 4). Yet, for many locations, only short periods of observations or hindcasts of tropical cyclones are available, which can be challenging for estimation of return values (corresponding to a period considerably longer than that for which data are available). For this purpose, a widely-used approach relies on the combination of synthetic cyclone track generation, wave modeling and extreme value analysis. The approach consists in the following steps: (1) tropical cyclones, extracted from either historical data (Knapp et al. 2010) or climate model simulations (Lin et al. 2012) are statistically resampled and modeled to generate synthetic, but realistic topical cyclone records. Based on a Monte Carlo approach (Emanuel et al. 2006; Vickery et al. 2000; Bloemendaal et al. 2020), a tropical cyclone dataset with same statistical characteristics as the input dataset, but spanning hundreds to thousands of years can then be generated; (2) for each synthetic cyclone, a hydrodynamic numerical model is used to compute the corresponding SWH over the whole domain of interest. An example of such simulator is the Global Tide and Surge Model of Bloemendaal et al. (2019); (3) SWH values at the desired coastal locations are extracted. Extreme value analysis (Coles et al. 2001) can then be used to estimate the corresponding return levels. As an illustration of the whole procedure, one can refer to the probabilistic hurricane-induced storm surge (including wave effects) hazard assessment performed by Krien et al. (2015) at Guadeloupe archipelago, Lesser Antilles.

Implementation of steps (1) and (2) can however be problematic. Generation of synthetic cyclones with realistic characteristics is a research topic in itself. Further, the hydrodynamic numerical model that can be prohibitively costly to execute. this might strongly limit the number of model runs (depending on the computational time cost), resulting in sparse, nonrepresentative data for extreme value modelling. To overcome this computational burden, possible solutions can either be based on parametric analytical models (like the ones used by Stephens and Ramsay 2014 in the Southwest Pacific Ocean) or on statistical predictive models (sometimes called meta- or surrogate models; Nadal-Caraballo et al. 2020). However, such approaches can only be considered "approximations". The former parametric analytical models introduce simplifying assump-

\footnotetext{
${ }^{2}$ https://data.jrc.ec.europa.eu/dataset/4f8c752b-3440-4e61-a48d-4d1d9311abfa
} 
tions regarding the physical processes involved. Statistical estimation is problematic, since inferences must be made concerning extreme quantiles of the distribution of quantities such as SWH, using a limited set of data.

\section{Objective and layout}

In the present work, we aim to tackle the problem of realistic return value estimation for small samples of tropical cyclones using a recently-developed procedure named STM-E, which has already been successfully applied in regions exposed to tropical cyclones near Japan (Wada et al. 2018) and in Gulf of Mexico (Wada et al. 2020). STM-E exploits all cyclone data drawn from a specific geographical region of interest, provided that certain modelling conditions are not violated by the data. This means in principle that STM-E provides less uncertain estimates of return values than statistical analysis of cyclone data at a single location. To date however, the STM-E methodology has not been directly validated: the objective of the present work is therefore to provide direct validation of return values (in terms of bias and variance characteristics, for return periods $T$ of hundreds of years) from STM-E analysis using sample data for modelling corresponding to a much shorter period $T_{0}(<T)$ of observation, drawn from a full synthetic cyclone database corresponding to a very long period $T_{L}\left(T_{L}>T\right)$ of observation.

In the following sections, we present a motivating application in the region of the Caribbean archipelago of Guadeloupe, for which synthetic cyclone data are available for a period $T_{L}$ corresponding to more that 3,000 years. We use the STM-E method to estimate the $T=500$-year return value for $\mathrm{SWH}$, and its uncertainty, based on random samples of tropical cyclones corresponding to $T_{0}=200$ years of observation. We compare estimates with empirical maxima from random samples corresponding to $T$ years of observation from the full synthetic cyclone data (covering $T_{L}$ years), and from standard extreme value estimates obtained using data (corresponding to $T_{0}$ years) from the specific location of interest only. Section 2 provides an outline of the motivating application. Section 3 describes the STM-E methodology. Section 4 presents the results of the application of STM-E to the region of main island pair (Basse-Terre and Grande-Terre) of Guadeloupe. Discussion and conclusions are provided in Section 5 .

\section{Motivating application}

75 The study area is located in a region of the Lesser Antilles (eastern Caribbean Sea) that is particularly exposed to cyclone risks (Jevrejeva et al. 2020) with several thousand fatalities reported since $1900^{3}$. We focus on the French overseas region of Guadeloupe, which is an archipelago located in the southern part of the Leeward Islands (see Figure 1).

This French overseas region has been impacted by several devastating cyclones in the past, including the 1776 event (of category 5 according to the Saffir-Simpson scale, Simpson and Saffir 1974) which led to $>6,000$ fatalities (Zahibo et al. 2007), and the "Great Hurricane" of 1928 (Desarthe 2015) with >1,200 fatalities; the latter was probably the most destructive tropical cyclone of the $20^{\text {th }}$ century. More recent destructive events include Hugo (in 1989, Koussoula-Bonneton 1994), and Maria (in 2017, which severely impacted Guadeloupe's banana plantations). The tracks of both Hugo and Maria are illustrated in Figure 1. Analysis of the HURDAT database (Landsea and Franklin 2013) reveals that approximately 0.6 cyclones per year passed

\footnotetext{
${ }^{3}$ http://www.emdat.be
} 

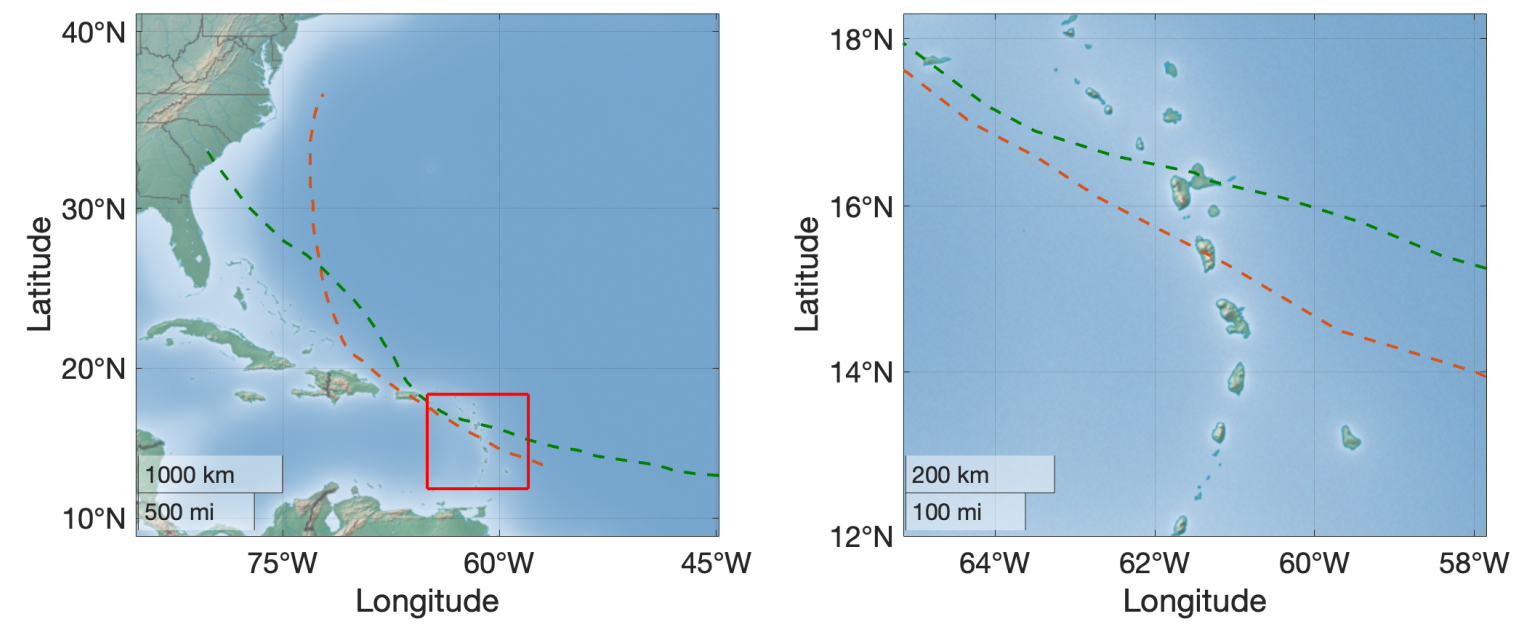

Figure 1. Region setting. Left: Full domain. The red rectangle indicates the region where the diagnostic of the STM-E approach is performed. The orange and green tracks respectively represent those of Maria (2017) and Hugo (1989) cyclones (data extracted from Landsea and Franklin (2013) with cyclone status "Hurricane"). Right: Enlarged view of the diagnostic region. The red rectangle indicates the region in the vicinity of Guadeloupe archipelago where the return levels are estimated.

within 400km of the study area on average for the period 1970-2019. Almost all events emanated from south east. More than $80 \%$ of the events passed close to the northern and eastern coasts of Guadeloupe's main island pair.

To assess the cyclone-induced storm surge hazard, Krien et al. (2015) set up a modelling chain similar to that described in the introduction: they randomly generate cyclonic events using the approach of Emanuel et al. (2006), and compute SWH and total water levels for each event over a wide computational domain (45-65W, 9.5-18.3N) using the ADCIRC-SWAN wave-current coupled numerical model. The interested reader can refer to Krien et al. (2015) for more implementation and validation detail.

In the present work, we use a total of 1971 synthetic cyclones passing nearby Guadeloupe (representative of 3,200 years, i.e. about 0.6 cyclone per annum) and the corresponding numerically calculated SWH. These results are used to derive empirically the 100-year SWH around the coast of Guadeloupe's main island pair for a smaller area of interest $\left(60.8-62.0^{\circ} \mathrm{W}, 15.8-\right.$ $16.6^{\circ} \mathrm{N}$; see Figure 2). These results are useful to assess flood risk at local scale, since they provide inputs of high resolution hydrodynamic simulations (see e.g. the use of wave over-topping simulations at La Reunion Island by Lecacheux et al. 2021). In the following, we analyse extreme SWH at 19 coastal locations around Guadeloupe's main island pair (on the 100m isodepth contour, see blue stars in Fig. 2), and at 12 locations along a line transect emanating to the north east from the island, corresponding to increasing water depth (see red triangles in Figure 2).

To illustrate the SWH data, Figure 3 depicts the spatial distributions of maximum SWH per location for the four cyclones with the largest single values of SWH in the whole synthetic cyclone database. All cyclones propagate from the south-east to the north-west with intense storm severity near the cyclone track, which reduces quickly away from the track. 


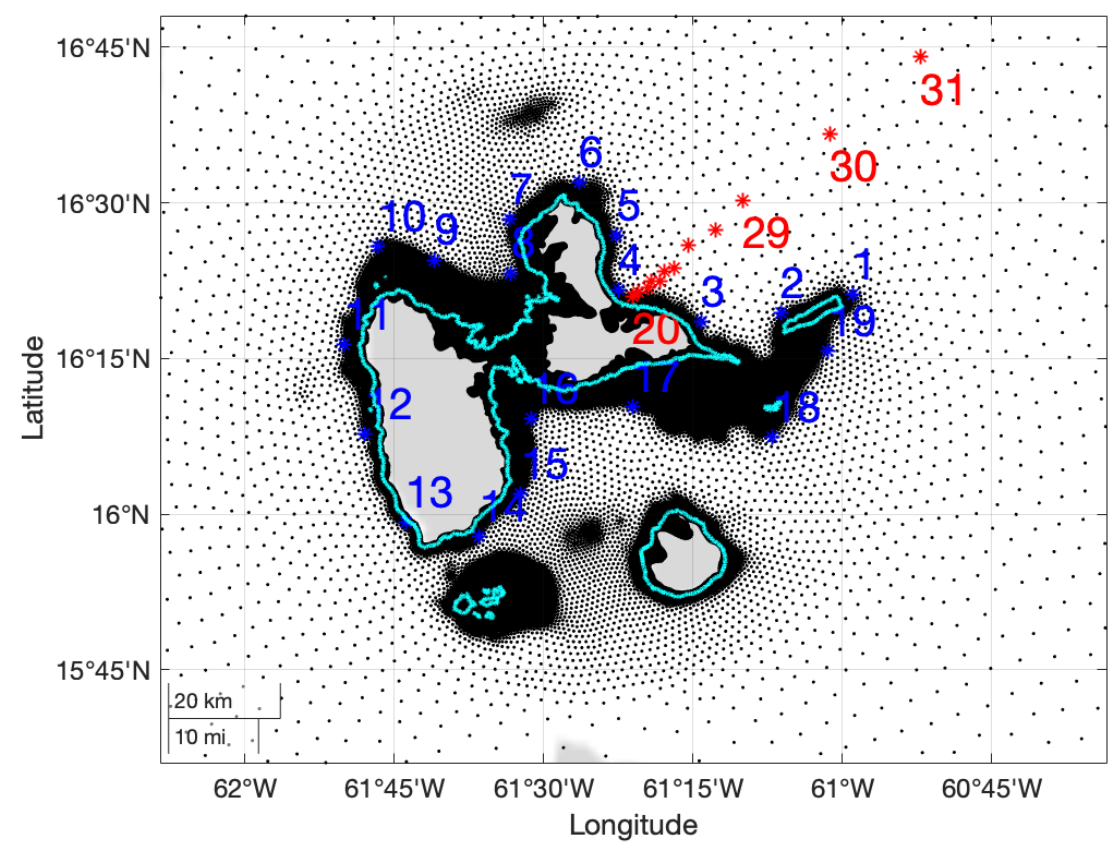

Figure 2. Illustration of Guadeloupe archipelago (administrative boundaries are outlined in light blue), showing calculation grid points (black dots), the selected locations along the iso-depth contour at $100 \mathrm{~m}$ (blue stars) and line transect (red triangles). Calculation grid points are more dense in shallow waters.

\section{Methodology}

In this section we describe the STM-E methodology used to estimate return values in the current work. Section 3.1 motivates the STM-E approach, and Section 3.2 outlines the modelling procedure. Section 3.3 provides a discussion of some of the diagnostic tests performed to ensure that STM-E modelling assumptions are satisfied.

\subsection{Motivating the STM-E model}

The STM-E procedure has been described in Wada et al. (2018) and Wada et al. (2020). The approach is intended to provide straightforward estimation of extreme environments over a spatial region, from a relatively small sample of rare events such as cyclones, the effects of any one of which do not typically influence the whole region. For each cyclone event, the spacetime characteristics of the event are summarised using two quantities, the space-time maximum (STM) of the cyclone and the spatial exposure (E) of each location in the region to the event. For any cyclone, the STM is defined as the largest value of SWH observed anywhere in the spatial region for the time period of the cyclone. The location exposure $\mathrm{E}$ is defined as the largest value of SWH observed at that location during the time period of the cyclone, expressed as a fraction of STM; thus values of $\mathrm{E}$ are in the interval $[0,1]$. 
https://doi.org/10.5194/nhess-2021-94

Preprint. Discussion started: 29 March 2021

(C) Author(s) 2021. CC BY 4.0 License.
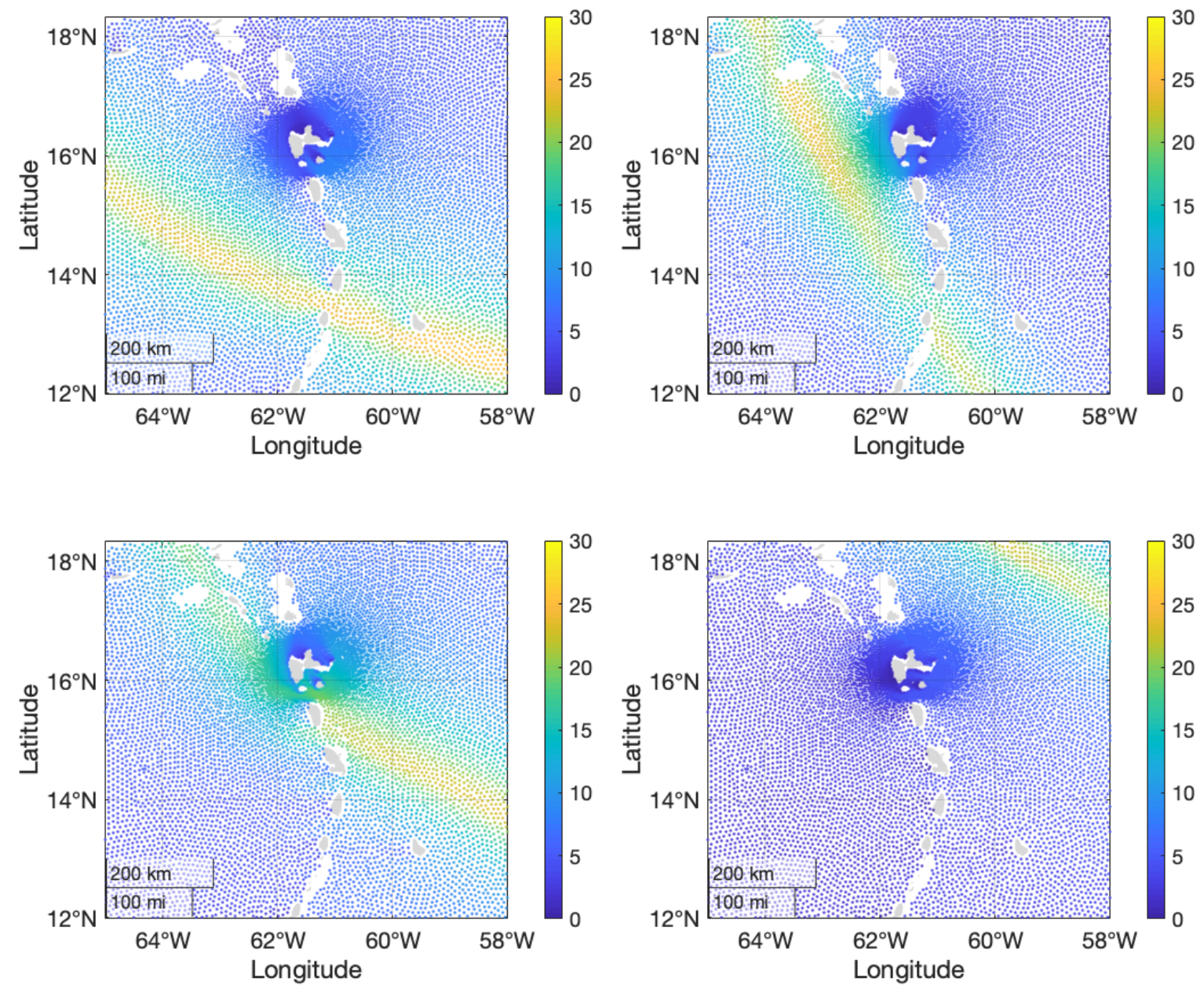

Figure 3. Spatial distributions of maximum SWH for the four largest synthetic cyclone events. Each panel gives the maximum SWH (over the period of the cyclone) per location.

The key modelling assumptions are then that (i) the future characteristics of STM and E over the region will be the same as those of STM and E during the period of observation, (ii) in future, at any location, it is valid to associate any simulated realisation of STM (under an extreme value model based on historical STM data) with any realisation of E (under on a model for the distribution of $\mathrm{E}$ based on historical $\mathrm{E}$ data). 


\subsection{STM-E procedure}

The steps of the modelling procedure are now described. The first three steps of the procedure involve isolation of data for the analysis. (a) An appropriate region of ocean is selected. The characteristics of this region need to be such that the underpinning conditions of the STM-E approach are satisfied (as discussed further in Section 3.3). (b) Then for each tropical cyclone event occurring in the region, the largest value of SWH observed anywhere in the region for the period of the cyclone (STM) is retained. (c) Next, per location in the region, the largest value of SWH observed during the period of the cyclone, expressed as a fraction of STM, is retained as the location exposure E to the cyclone.

The next three steps of the analysis involve statistical modelling and simulation. (d) First, an extreme value model is estimated using the largest values from the sample of STM; typically, a generalised Pareto distribution (see e.g. Coles et al. 2001) is assumed. Then a model for the distribution of location exposure $\mathrm{E}$ is sought; typically we simply re-sample at random with replacement from the values of historical $\mathrm{E}$ for the location, although model-fitting is also possible. (e) Next, realisations of random occurrences of STM from (d), each combined with a randomly-sampled exposure E per location, permits estimation of the spatial distribution of SWH corresponding to return periods of arbitrary length. (f) Finally, diagnostic tools are used to confirm the consistency of simulations (e) under the model with historical cyclone characteristics.

\subsection{Diagnostics for STM-E modelling assumptions}

The success of the current approach relies critically on our ability to show that simplifying assumptions regarding the characteristics of STM and exposure are justified for the data to hand. In particular, the approach assumes that (i) the distribution STM does not depend on cyclone track, environmental covariates, space and time, and (ii) the distribution of exposure per location does not depend on STM, cyclone track, environmental covariates and time. Diagnostic tests are undertaken to examine the plausibility of these conditions for region of ocean of interest for each application undertaken. Establishing the validity of the STM-E conditions is vital for credible estimation of return value estimates. Section 5 of Wada et al. (2018) provides a detailed discussion of some of the diagnostic tests that should be considered to judge that the STM-E conditions are non violated in any particular application.

The absence of spatial trend in STM over the region can be assessed by estimating the linear or rank correlation between STM values along longitude-latitude transects with arbitrary orientation in the region. For comparison, the "null" distribution of correlation can be estimated using random permutations of the STM values.

Return value estimates from STM-E are also potentially sensitive to the choice of region for analysis. We assume that the extremal behaviour of STM can be considered homogeneous in the region, suggesting that the region should sufficiently small that the same physics is active throughout it. However, the region also needs to contain sufficient evidence for cyclone events and their characteristics to allow reasonable estimation of tails of distributions for SWH per location. The absence of dependence between STM and E per location can be assessed by calculating the rank correlation between STM $S$ (a space-time maximum) and exposure $E_{j}$ (at location $j$ ) using Kendall's tau statistic. If the values of $S$ and $E_{j}$ increase together, the value of Kendall's tau statistic will be near to unity. If there is no particular relationship between $S$ and $E_{j}$, the value of Kendall's tau 
will be near zero. For large $n$, if $S$ and $E_{j}$ are independent, the value of Kendall's tau is approximately Gaussian-distributed with zero mean and known variance, providing a means of identifying unusual values which may indicate dependence between $S$ and $E_{j}$. An illustrative spatial plot of Kendall's tau is given in Section 4.

Finally, estimates from STM-E are potentially sensitive to the extreme value threshold $\psi_{n}$ (or equivalently the sample size $n$ of largest observations of STM) chosen to estimate the tail of the distribution of STM over the region. Results in Section 4 are reported for a number of choices of $n$ for this reason.

\subsection{Modelling STM and estimating return values}

Suppose we have isolated a set of $n_{0}$ values of STM using the procedure above. We use the largest $n \leq n_{0}$ values $\left\{s_{i}\right\}_{i=1}^{n}$, corresponding to exceedances of threshold $\psi_{n}$, to estimate a generalised Pareto model for STM, with probability density function

$$
\begin{aligned}
& \operatorname{Pr}\left(S \leq s \mid S>\psi_{n}\right)=F_{S \mid \psi_{S}}(s) \\
& \stackrel{\stackrel{\operatorname{large}}{\approx}_{n} F_{G P}(s)}{ }=1-\left(1+\frac{\xi}{\sigma_{n}}\left(s-\psi_{n}\right)\right)^{-1 / \xi} \text { for } \xi \neq 0 \\
&=1-\exp \left(-\frac{1}{\sigma_{n}}\left(s-\psi_{n}\right)\right) \text { otherwise }
\end{aligned}
$$

with shape parameter $\xi \in \mathbb{R}$ and scale parameter $\sigma_{n}>0$. Choice of $n$ is important, to ensure reasonable model fit and biasvariance trade-off. The estimated value of $\xi$ should be approximately constant as a function of $n$ for sufficiently small $n$. The full distribution $F_{S}(s)$ of STM can then be estimated using

$F_{S}(s)=\left\{\begin{array}{l}F_{n}^{*}(s) \text { for } s \leq \psi_{n} \\ \tau_{n}+\left(1-\tau_{n}\right) F_{S \mid \psi_{n}}(s) \text { otherwise }\end{array}\right.$

where $F_{n}^{*}(s)$ is an empirical "counting" estimate below threshold $\psi_{n}$, and $\tau_{n}$ is the non-exceedance probability corresponding the $\psi_{n}$, again estimated empirically.

170 Using this model, we can simulate future values $H_{j}$ of SWH at any location $j,(j=1,2, \ldots, p)$ in the region, relatively straightforwardly. Suppose that $E_{j}$ is the location exposure at location $j$, and $F_{E_{j}}$ its cumulative distribution function, estimated empirically. Since then $H_{j}=E_{j} \times S$, the cumulative distribution function of $H_{j}$ can be estimated using

$$
\begin{aligned}
F_{H_{j}}(h) & =\mathbb{P}\left(H_{j} \leq h\right) \\
& =\int_{s} \mathbb{P}\left(E_{j} S \leq h \mid S=s\right) f_{S}(s) d s \\
& =\int_{s} \mathbb{P}\left(E_{j} \leq h / s\right) f_{S}(s) d s \\
& =\int_{s} F_{E_{j}}(h / s) f_{S}(s) d s
\end{aligned}
$$

where $f_{S}(s)$ is the probability density function of STM, corresponding to cumulative distribution function $F_{S}(s)$ estimated in Equation 2. 


\section{Application of STM-E to cyclones SWH near Guadeloupe}

The STM-E methodology outlined in Section 3 is applied to data for the neighbourhood of Guadeloupe's main island pair described in Section 2. The objective of the analysis is to estimate the $T=500$-year return level for $\mathrm{SWH}$ from $T_{0}=200$ years of data. First, details of the set-up of the STM-E analysis are provided in Section 4.1. Then, in Section 4.2, we describe two competitor methods included for comparison with STM-E. Section 4.3 the describes estimates for the 500-year return value on the $100 \mathrm{~m}$ iso-depth contour around Guadeloupe's main island pair and the line transect introduced in Section 2, illustrated in Figure 2, using maximum likelihood estimation (see e.g. Hosking and Wallis 1987, Davison 2003). For comparison, Section 4.4 then provides estimates obtained using probability weighted moments (see e.g. Furrer and Naveau 2007,de Zea Bermudez and Kotz 2010a, de Zea Bermudez and Kotz 2010b). Section 4.5 describes some of the diagnostic tests undertaken to confirm that the fitted model is reasonable.

\subsection{Details of STM-E application}

The spatial region of interest is the neighbourhood of Guadeloupe's main island pair in the Caribbean Sea, corresponding to approximately longitudes $12^{\circ}-18^{\circ} \mathrm{N}$ and latitudes $58^{\circ}-65^{\circ} \mathrm{W}$ (see Figure 1). An initial analysis using Kendall's tau suggests the full region $\left(45^{\circ}-65^{\circ} \mathrm{W}, 9.5^{\circ}-8.3^{\circ} \mathrm{N}\right)$ shows dependency of STM and exposure, with stronger cyclones tending to pass through the western part of the region. However, if a very high threshold $\psi \approx 20 \mathrm{~m}$ was selected for analysis, reasonable decoupling of STM and E could be achieved, with relatively less intense tropical cyclones neglected. Since the focus of the current work is the ocean environment of Guadeloupe archipelago, a smaller region (see Figure 1, right panel) was defined. For this region, Kendall's tau indicated low dependence between STM and E for thresholds $\psi$ of 10m and above, as illustrated in the left panel of Figure 4.

The right panel of Figure 4 shows the location and magnitude of STM for each of the $n=60$ largest cyclones observed in the region. There is no obviously no spatial dependence between the size of STM and its location. In Section 3.3, we discuss the use of rank correlation of STM along latitude-longitude transects as a means to quantify dependence in general. In fact, the Kendall's tau analysis illustrated in the left panel would also indicate any strong spatial dependence in STM; therefore, results of the rank correlation analysis are not presented. We conclude that Figure 4 does not suggest that the modelling assumptions underlying STM-E are not satisfied.

The relatively large number of boundary STM values reflect occurrences of cyclones, the true STM locations of which occur outside the analysis region. For these events, the value of STM used for analysis is the largest value of SWH observed within the analysis region.

Specific interest lies in the variation of extreme return value around Guadeloupe, and the rate of increase of return value with increasing water depth away from the coasts. For this reason, two sets of locations were adopted for details analysis, reported here. The first set corresponds to 19 locations on an approximately iso-depth contour at 100m depth around the main island pair of Guadeloupe. This depth value is typically chosen to define the boundaries of the local scale high resolution flooding simulations. The second set corresponds to 12 locations on a line transect emerging approximately normally from the north- 

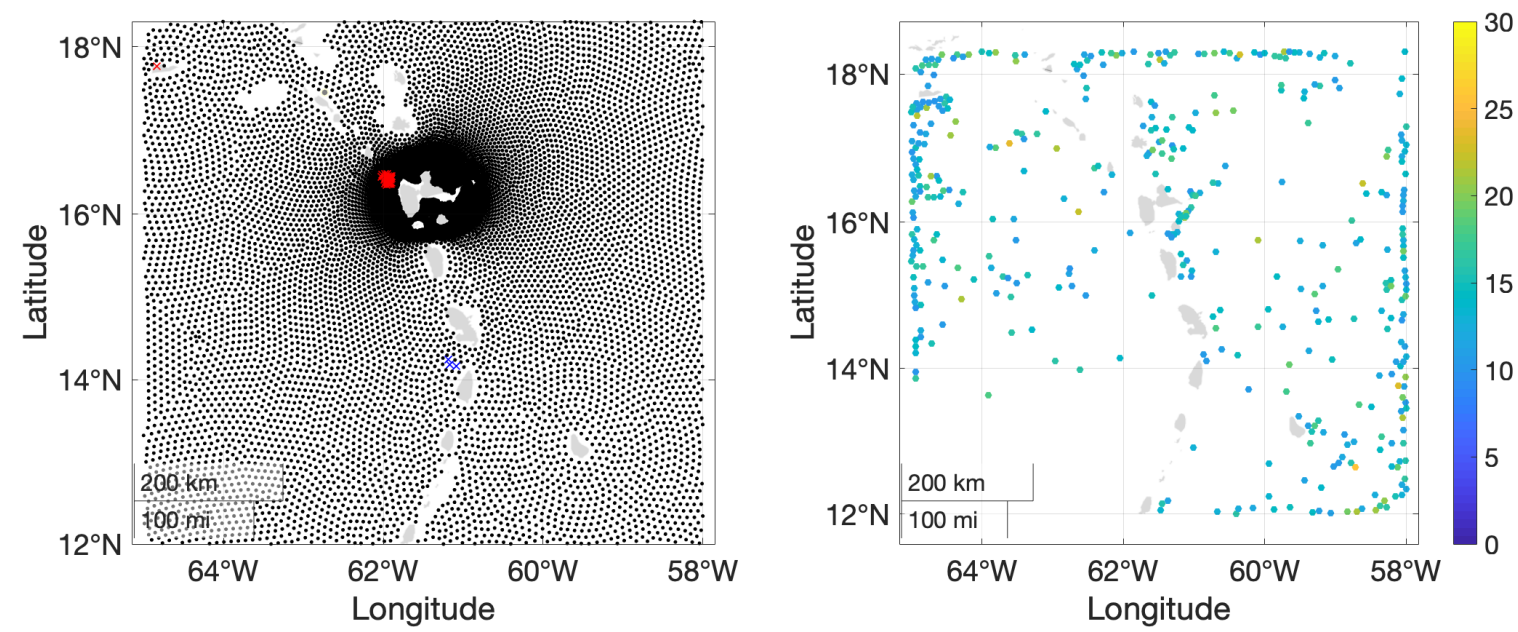

Figure 4. Diagnostics for STM-E. Left: plot of Kendall's tau for analysis region using a threshold of 10m. Each point corresponds to a location where SWH data is available and water depth exceeds $100 \mathrm{~m}$. The black dots indicate values of Kendall's tau within the $90 \%$ confidence interval. Red (blue) cross indicate positive (negative) values of Kendall's tau exceeding the $90 \%$ confidence band. The percentage of recorded exceedances of the $90 \%$ confidence band for Kendall's tau is less than $10 \%$. Right: Locations of all STMs exceeding 10m coloured by size of STM in metres.

east of the main island pair of Guadeloupe. We focus on the north-east part, because it is where the exposure to cyclones is the highest. The contour and transect are illustrated in Figure 2, and location numbers are listed.

Focus of the analysis is estimation of the $T=500$-year return value for SWH on the iso-depth contour and line transect, based on $T_{0}=200$ years of data, to quantify the uncertainty in the 500-year return value using STM-E analysis, the following procedure based on random sub-sampling was adopted. For STM-E analysis, the following procedure was adopted. (a) Randomly select the appropriate number of cyclones (corresponding to $T_{0}$ years of observation) from the $T_{L}$ years of synthetic cyclones. (b) Identify the largest $n$ values of STM in the sample, for $n=20,30,40,50$ and 60. (c) Estimate a model for the distribution of STM using maximum likelihood estimation or the method of probability weighted moments. (d) Estimate the empirical distribution of exposure E per location on the iso-depth contour and line transect. (e) Estimate the 500-year return value as the quantile of the distribution $F_{H_{j}}$ of significant wave height at location $j$ with non-exceedance probability $1-\left(T_{0} / n\right) / T$. Finally, the whole procedure (a)-(e) is repeated 100 times to quantify the uncertainty in the $T$-year return value.

Figure 5 illustrates the tails of the distribution of STM from the largest 30 values of STM from each of 100 random samples corresponding to 200 years, and from the full sample of synthetic cyclones. It can be seen that the 500 -year return value for STM lies in the region $(20,30) \mathrm{m}$. Typical distributions of exposure E per location are given in Figure 11, and discussed in due course. 


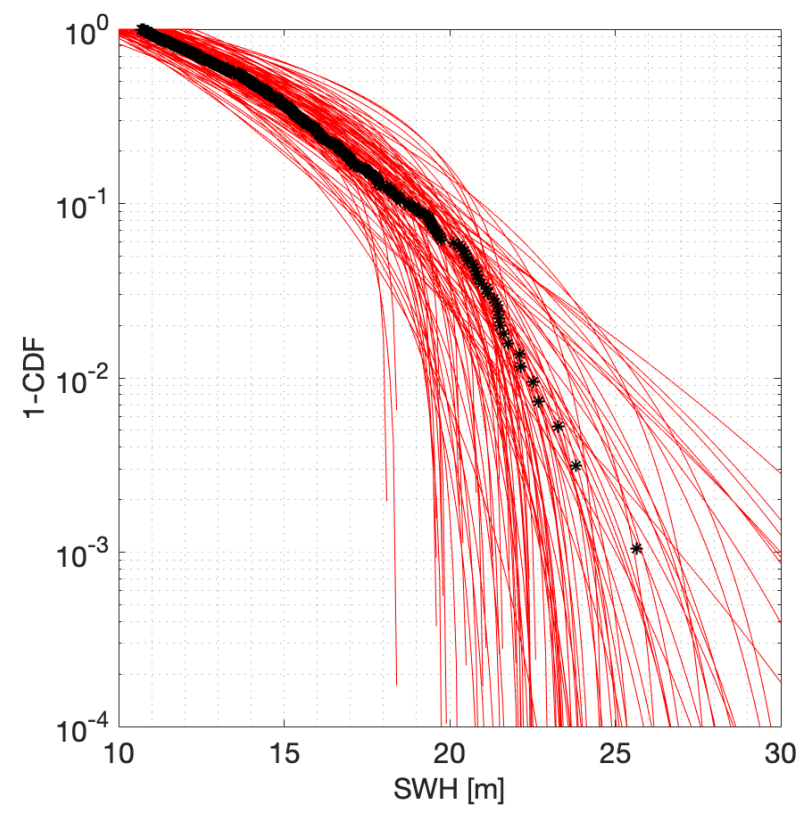

Figure 5. Variability of tail of distributions for STM on log scale. Each of the 100 red lines is estimated from a sample size 30 of largest STM values from a random sample of 124 cyclones corresponding to 200 years of observation. The black points indicate the corresponding empirical distribution of STM from the full synthetic cyclone data.

\subsection{Benchmarking against the full cyclone database and single-location analysis}

One important advantage of the current synthetic cyclone database is that it corresponds to long time period relative of $T_{F}=$ 3,200 years, much longer than the return period of $T=500$ years being estimated in the current analysis. Thus, we are able to estimate the 500-year return value at any location using the full synthetic cyclone data, by simply sampling 310 cyclones (corresponding to 500 years, with annual cyclone occurrence rate of 0.62 ) at random. This provides a direct empirical estimate.

From previous work, a key advantage found using the STM-E approach is that it provides less uncertain estimates at a location compared with conventional "single location" analysis. We wish to demonstrate in the current work that this is also the case. For this reason, we also calculate estimates for comparison with those from STM-E, based on independent analysis of cyclone data from each location of interest. The procedure is as follows. (a) Randomly select the appropriate number of cyclones (corresponding to $T_{0}$ years) from the $T_{F}$ years of synthetic cyclones for a single location (indexed by $j$ ). (b) Identify the largest value of SWH per cyclone, and call this the peak SWH. (c) Identify the $n$ values of peak cyclone SWH in the sample, for $n=20,30,40,50$ and 60. (d) Estimate a model for the distribution of peak SWH using maximum likelihood estimation or the method of probability weighted moments. (e) Estimate the $T$-year return value as the quantile of the distribution of peak significant wave at location $j$ with non-exceedance probability $1-\left(T_{0} / n\right) / T$. Finally, repeat (a)-(e) 100 times to quantify the uncertainty in the $T$-year return value. 
https://doi.org/10.5194/nhess-2021-94

Preprint. Discussion started: 29 March 2021

(c) Author(s) 2021. CC BY 4.0 License.

\subsection{Maximum likelihood estimation}

Figure 6 illustrates the 500-year return value for SWH using maximum likelihood estimation for locations on the $100 \mathrm{~m}$ isodepth contour around Guadeloupe's main island pair, with location numbers given in Figure 2. The figure caption gives relevant details of the figure layout. Across the 19 locations considered, the 500-year return value is estimated using STM-E (blue), single-location (red) and full synthetic cyclone data (black); in general, there is good agreement between estimates per location. It can be seen however (from the longer red whiskers) that the uncertainty in single-location estimates is greater than estimated from the full data and from STM-E. In general, the uncertainty in return value estimated using STM-E more closely reflects that seen in the full synthetic cyclone data.

Per location, as the number points used for STM-E estimation increases, there is evidence for reduction in the uncertainty with which the return value is estimated, as might be expected. However, there is also some evidence for a small increase in the mean estimated return value. This is explored further in Section 4.5. There is no corresponding evidence for reduced uncertainty in the single-location analysis. There are more outlying estimates of return value for single-location analysis than for STM-E.

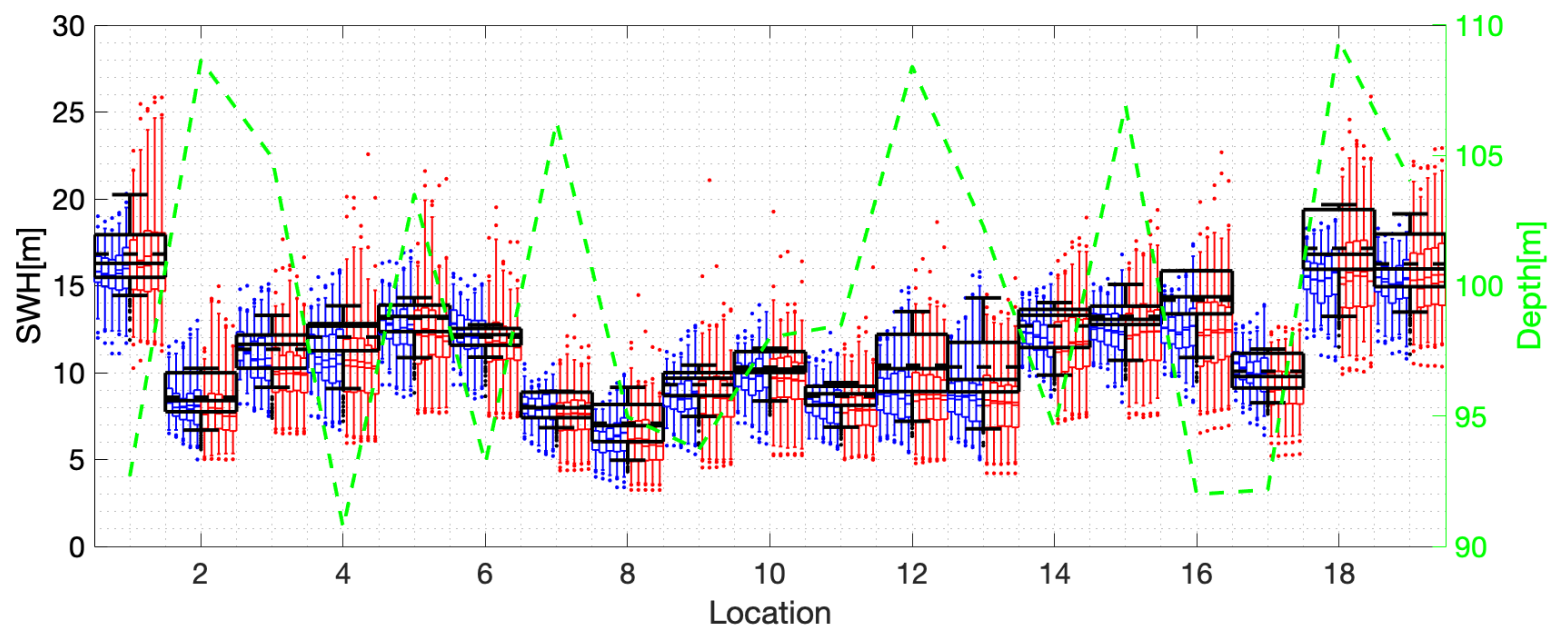

Figure 6. 500-year return value for SWH using maximum likelihood estimation for the $100 \mathrm{~m}$ iso-depth contour. The $\mathrm{x}$-axis gives the reference numbers of the 19 locations on the contour. Location numbers are given in Figure 2. Corresponding to each location, the blue box-whiskers summarise the estimated return value from STM-E for different sample sizes 20, 30, 4050 and 60; the red box-whiskers summarise the estimated return values from single-location analysis for different sample sizes. For each blue-red cluster of box-whiskers corresponding to a specific location, return value estimates for the increasing sequence of sample sizes are plotted sequentially outwards from the centre of the cluster. The black box-whisker for each location corresponds to the empirical estimate of the return value obtained directly from the synthetic cyclone data. For all box-whiskers, the box represents the inter-quartile interval, the median and mean are shown are solid and dashed lines. Whiskers represent the $2.5 \%$ to $97.5 \%$ interval. Exceedances of this $95 \%$ interval are shown as dots. The green dashed line and the right-hand $\mathrm{y}$-axis give the water depth at each location. 

general characteristics of this figure are similar to those of Figure 6. The return value increases as would be expected with increasing water depth. Single-point estimates are more variable that those from STM-E. There is again some evidence that the STM-E median estimate increases with increasing sample size, but this trend is small compared to the uncertainty in the return value estimated empirically from the full synthetic cyclone data set. We infer from the analysis that water depth has little effect on the performance of the STM-E approach.

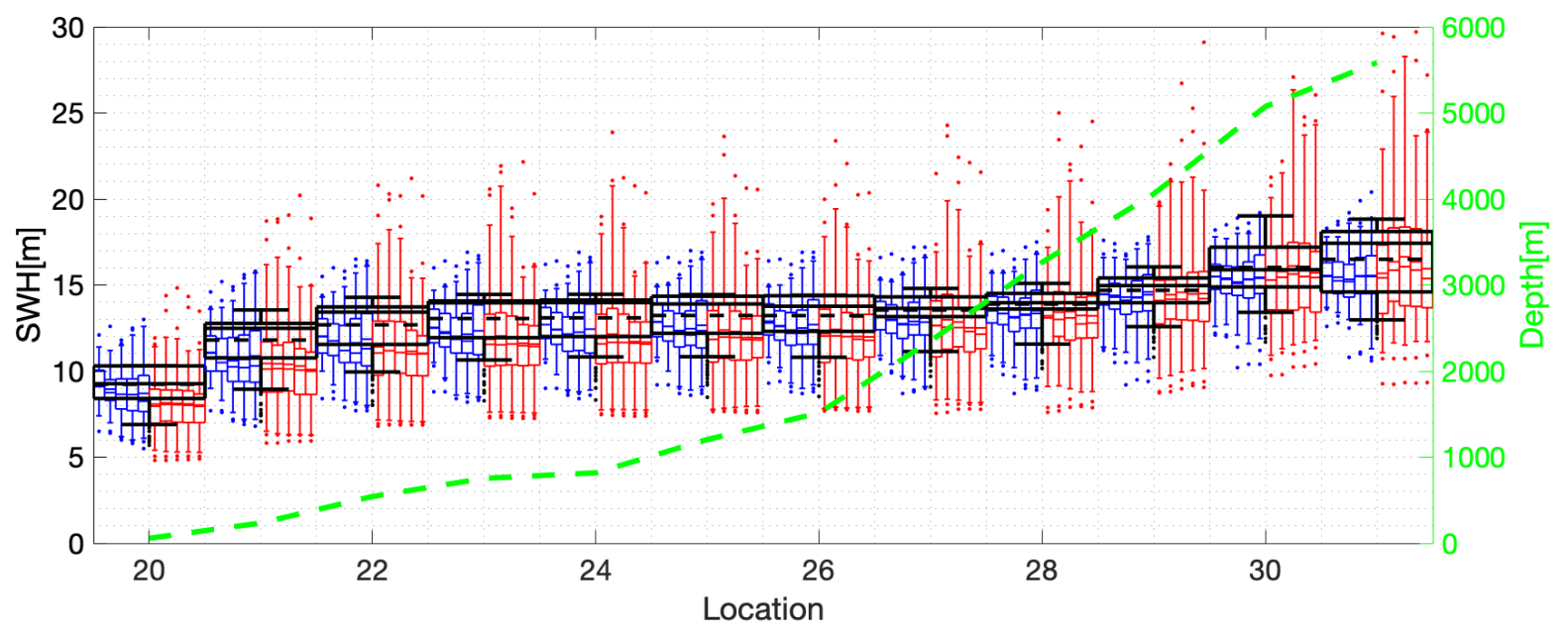

Figure 7. 500-year return value for SWH using maximum likelihood estimation for the line transect. The x-axis gives the reference numbers of the 12 locations on the transect. Briefly, for each location, blue and red box-whiskers summarise the estimated return value from STM-E and single-location analysis respectively; see caption of Figure 6 for other details. The black box-whisker plot per location corresponds to the empirical estimate of the return value obtained directly from the synthetic cyclone data. The green dashed line and the right-hand y-axis give the water depth at each location.

\subsection{Results estimated using probability weighted moments}

Estimates for the 500-year return value on the iso-depth contour, obtained using the method of probability weighted moments, are shown in Figure 8. The behaviour of STM-E and single-location estimates shown is very similar to that illustrate for maximum likelihood estimation in Figure 6. There is some evidence that both STM-E and single-location estimates obtained using probability weighted moments show somewhat lower bias. Again, this is perhaps not surprising given the relative characteristics of maximum likelihood and probability weighted moment estimators for small samples. This is further discussed in Section 4.5.

Results for the line transect using probability weighted moments are given in Figure 9. Again, the figure shows similar trends to Figure 7, except perhaps for a slightly lower bias in estimates using probability weighted moments. 


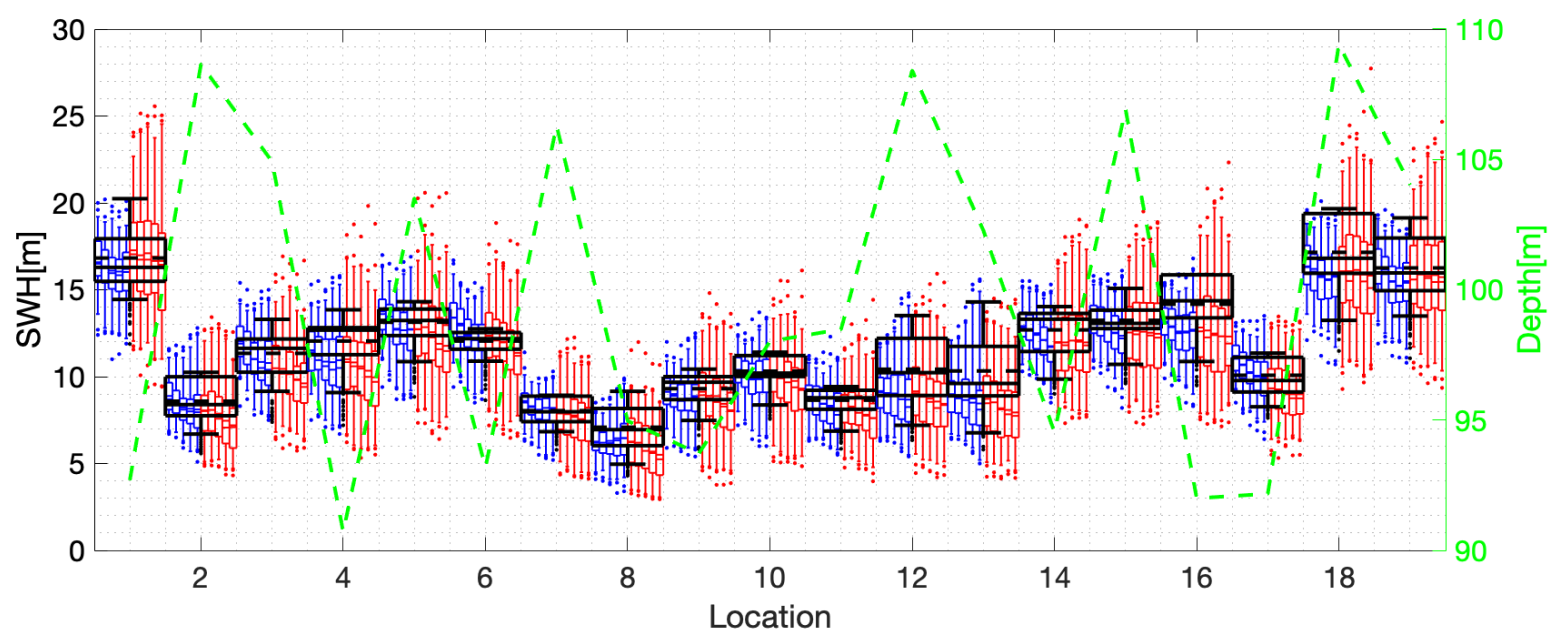

Figure 8. 500-year return value for SWH estimated using probability weighted moments for the 100m depth contour. The $\mathrm{x}$-axis gives the reference numbers of the 19 locations on the contour. Briefly, for each location, blue and red box-whiskers summarise the estimated return value from STM-E and single-location analysis respectively; see caption of Figure 6 for other details. The black box-whisker plot per location corresponds to the empirical estimate of the return value obtained directly from the synthetic cyclone data. The green dashed line and the right-hand $\mathrm{y}$-axis give the water depth at each location.

\subsection{Assessment of model performance}

Results from Sections 4.3 and 4.4 suggest that there is a small increasing trend in return value estimates from STM-E as a function of increasing sample size for inference. Results also suggest that the method of probability weighted moments gives less bias in estimates for return values. We investigate these effects further here. Figure 10 gives estimates for the 500-year return value of space-time maximum STM (as opposed to the full STM-E estimate) as a function of sample size used for estimation, using maximum likelihood estimation (upper panel) and probability weighted moments. Also shown (in red, at zero on the X-axis) is the empirical estimate of the 500-year STM return value obtained directly from the synthetic cyclone data. The figure shows a number of interesting effects. Firstly, maximum likelihood estimates are in this case biased low, and the extent of the bias reduces with increasing sample size $n$. It should be noted, however, that the extent of the bias is small relative to the uncertainty in the the return value. Secondly, the method of probability weighted moments is less biased for small sample sizes. But interestingly, the mean (or median) return value estimate also appears to increase with sample size, so that at a sample size of 60, probability weighted moment estimates are clearly biased high in this example. Further, uncertainties on return value estimates from probability weighted moments are larger than those from maximum likelihood estimation.

There are many studies in the literature comparing the performance of different methods of estimation of extreme value models. The method of probability weighted moments is known to perform relatively well relative to maximum likelihood estimation for small samples (see, e.g., Jonathan et al. (2021), Section 7 for a discussion). For small samples, for example, 


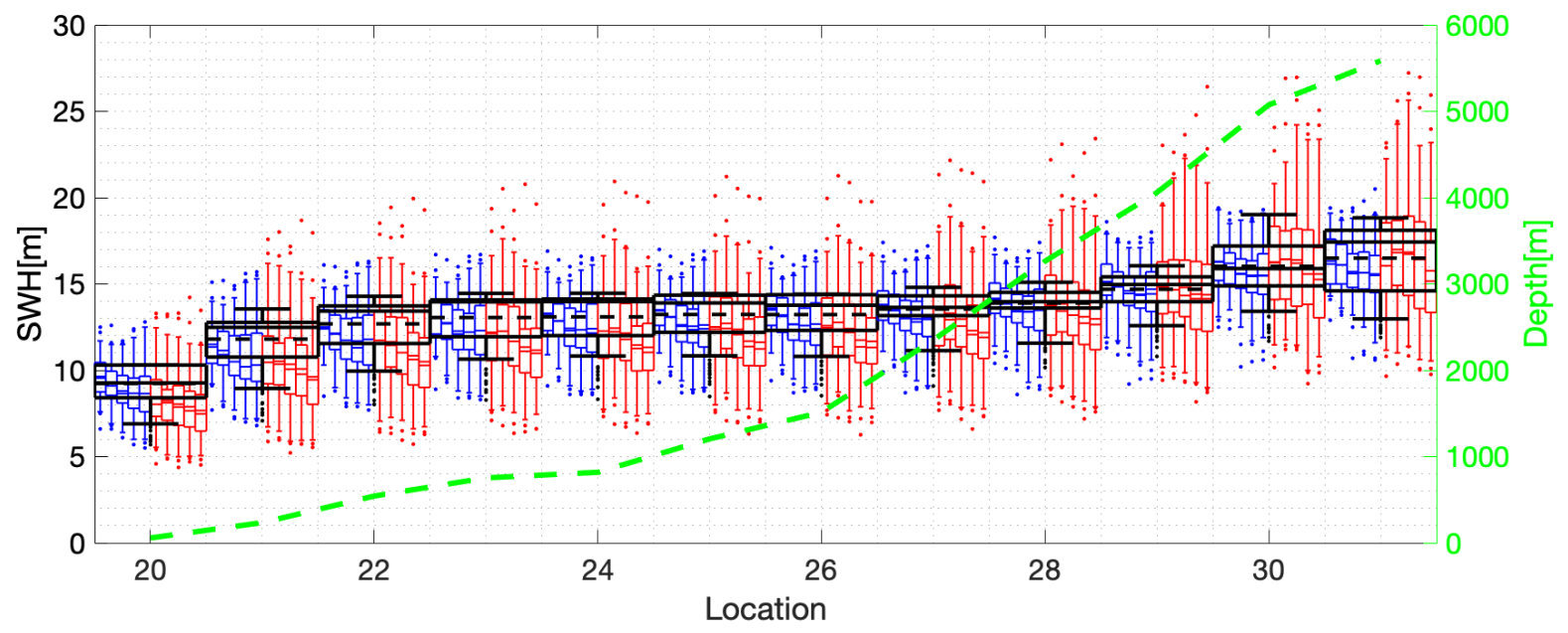

Figure 9. 500-year return value for SWH estimated using probability weighted moments for the line transect. The $\mathrm{x}$-axis gives the reference numbers of the 12 locations on the transect. Briefly, for each location, blue and red box-whiskers summarise the estimated return value from STM-E and single-location analysis respectively; see caption of Figure 6 for other details. The black box-whisker plot per location corresponds to the empirical estimate of the return value obtained directly from the synthetic cyclone data. The green dashed line and the right-hand $\mathrm{y}$-axis give the water depth at each location.

maximum likelihood estimation is known to underestimate the generalised Pareto shape parameter, and over-estimate the corresponding scale parameter, leading to bias in return value estimates. Given these facts, we do not consider the small trends shown in the current analysis to be particularly interesting, or of particular concern. However, the trends do illustrate the importance of performing an extreme value analysis with great care, particularly for small sample sizes.

One of the assumptions underpinning the STM-E approach is that the exposure distribution at a location is not dependent on the magnitude of STM. We investigate this effect in Figure 11. The figure gives exposure curves for each of the 100 random data samples of 200 years used to estimate the STM-E model and return value characteristics in this section, for location 10 (see Figure 2). Panels correspond to different sample sizes. We are concerned in particular that there are no patterns in exposure distribution related to the size of STM for the corresponding data sample. For this reason, each panel also shows the exposure distribution corresponding to the data sample with largest and smallest STM in red and blue. It can be seen that the coloured curves are not obviously unusual with respect to the other curves. Similar plots for other locations also do not give cause for concern.

\section{Discussion}

This work considers the estimation of $P$-year return values for SWH over a geographic region, from small sets of $T_{0}$ years of synthetic tropical cyclone data, using the STM-E (space-time maxima and exposure) methodology. We assess the methodology 


\section{(a) MLE}

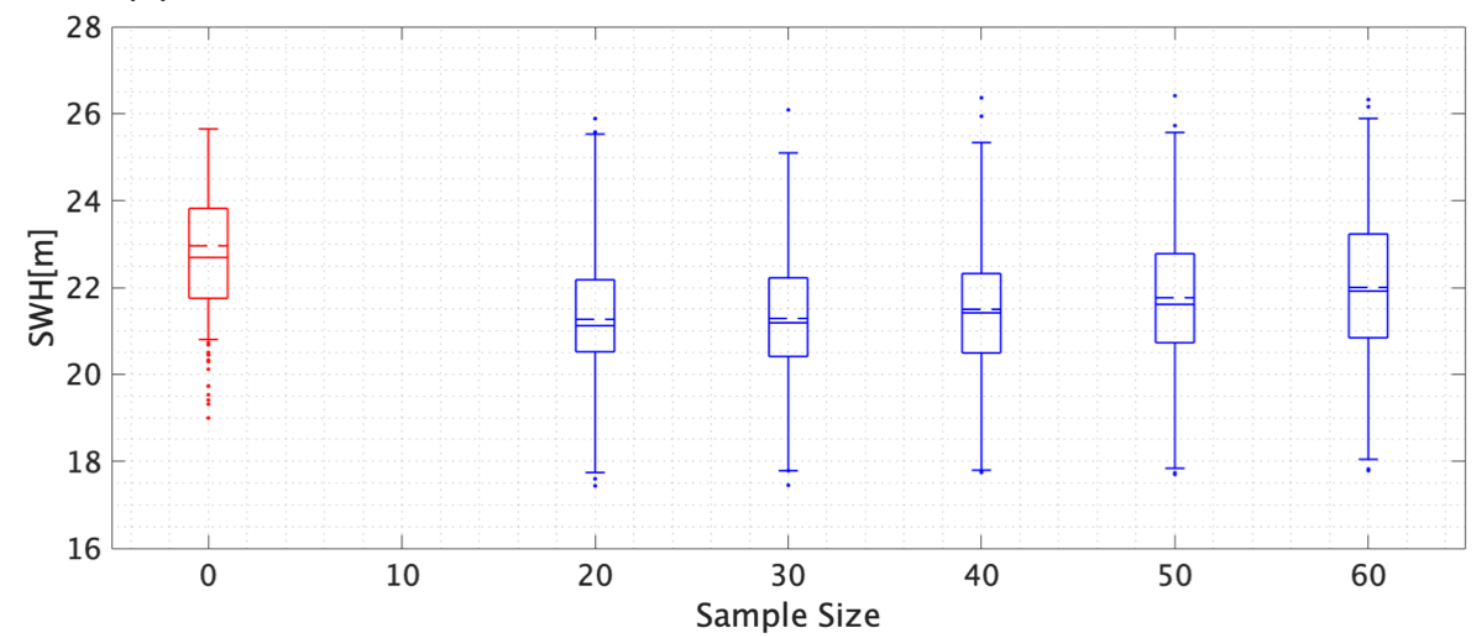

\section{(b) PWM}

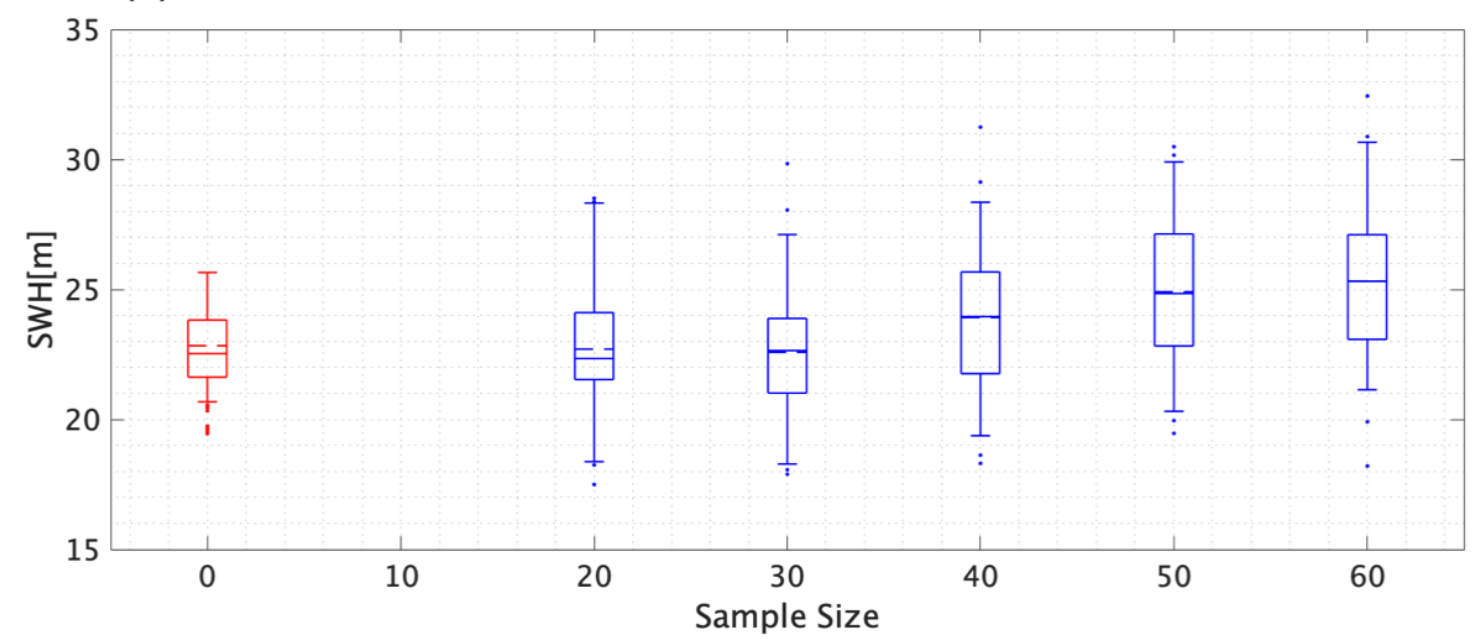

Figure 10. The effect of sample size on estimates of return values for STM. Blue box-whiskers give estimates for the 500-year STM return value using different sample sizes based on maximum likelihood estimation (upper panel) and the method of probability weighted moments (lower panel). The red box-whisker in each panel gives an empirical estimate of the same return value obtained directly from the synthetic cyclone data.

by comparing estimates of the $T$-year return value $\left(T>T_{0}\right)$ for locations in the region from STM-E, with those estimated directly from a large database corresponding to $T_{L}(>T)$ years of synthetic cyclones. We find that STM-E performs well in terms of bias and uncertainty of $T=500$-year return value from $T_{0}=200$ years of data in the region of Guadeloupe archipelago. We also compare STM-E estimates of $T$-year return values for locations in the region with those obtained by 
https://doi.org/10.5194/nhess-2021-94

Preprint. Discussion started: 29 March 2021

(c) Author(s) 2021. CC BY 4.0 License.
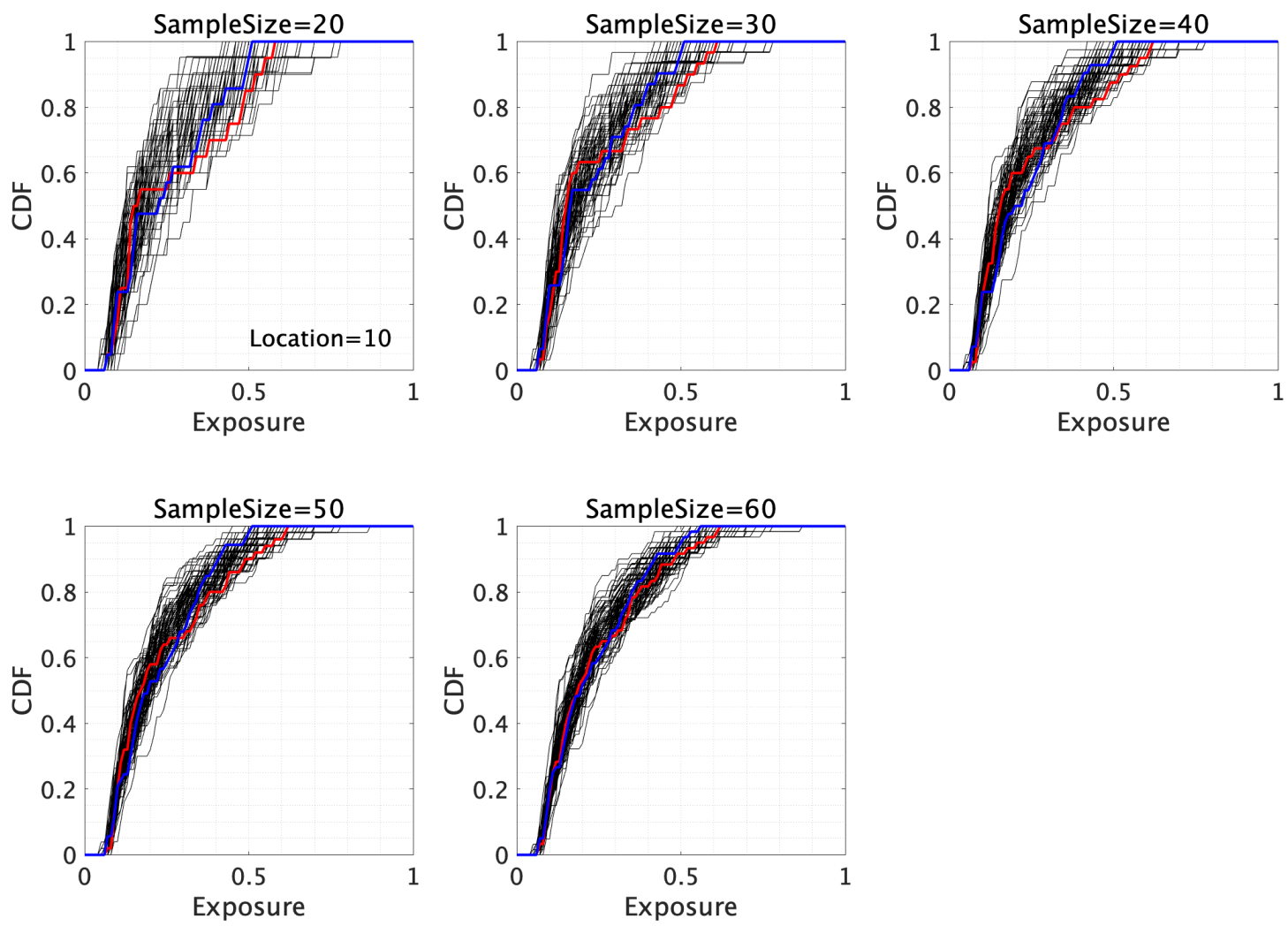

Figure 11. Exposure distributions for location 10. Each curve drawn is an empirical cumulative distribution function of exposure for a cyclone at location 10, estimated for one of 100 random data samples corresponding to 200 years. The red curve indicates the exposure distribution for the data sample with maximum STM, and the blue curve for the data sample with minimum STM. Panels represent different STM-E sample sizes.

extreme value analysis of data (for $T_{0}$ years) at individual locations. We find that STM-E provides more realistic estimates of return value uncertainty.

For reasonable application of the STM-E approach, it is important that characteristics of tropical cyclones over the region under consideration satisfy a number of simplifying conditions. These conditions are shown not to be violated for a region around Guadeloupe archipelago, but that use of the STM-E method over a larger spatial domain would not be valid (see e.g.

310 Wada et al. 2019). This demonstrates that selection of an appropriate geographical region for STM-E analysis is critical to its success. Once such a region is specified, we find that the space-time maxima and exposure (STM-E) method provides a simple but principled approach to return value estimation within the region from small samples of tropical cyclone data. 
Return value estimates from STM (see e.g. Figure 10) show a small increasing bias with increasing sample size $n$ for extreme value estimation. However, the resulting bias in full STM-E return values is small, especially compared with the inherent variability of the return value estimate. Corresponding estimates based on single-location analysis also show relatively small but increasing negative bias with increasing sample size. In the present work, the tail of the distribution of STM was estimated by fitting a generalised Pareto model, using either maximum likelihood estimation or the method of probability weighted moments. Estimates for extreme quantiles of STM using either approach are in good agreement.

Table 1 summarises the performance of STM-E and single-location analysis in estimation of the bias and uncertainty of the 500 -year return value, relative to empirical estimates from the full synthetic cyclone data, for analysis sample sizes of $n=20,30$, 40, 50 and 60. Bias $B(n ; \mathrm{Mth})$ and uncertainty $U(n ; \mathrm{Mth})$ are estimated as average characteristics over all $|\mathcal{L}|=31$ locations $\ell \in \mathcal{L}$ on the iso-depth contour and line transect corresponding to the relevant sample size, using the expressions

$$
B(n ; \text { Mth })=\frac{1}{|\mathcal{L}|} \sum_{\ell \in \mathcal{L}}\left(\tilde{h}(\ell ; n, \text { Mth })-\tilde{h}_{0}(\ell ; n)\right), \quad U(n ; \text { Mth })=\frac{1}{|\mathcal{L}|} \sum_{\ell \in \mathcal{L}}\left(\frac{r(\ell ; n, \text { Mth })}{r_{0}(\ell ; n)}-1\right) .
$$

Here, $\tilde{h}(\ell ; n, \mathrm{Mth})$ and $\tilde{h}_{0}(n)$ correspond to the median 500-year return value estimated using sample size $n$ from inference method Mth (either maximum likelihood or probability-weighted moments) and directly from the full synthetic cyclone database; $r(\ell ; n, \mathrm{Mth})$ and $r_{0}(n)$ are the corresponding $95 \%$ uncertainty bands. Zero-values for $B$ and $U$ correspond to perfect agreement between model-based estimates and their full database equivalents. The table summarises the findings presented pictorially in Figures 6-8. In terms of bias, STM-E and single-location estimates underestimate the return value on average. STM-E is less biased than single location estimates except for sample sizes 20 and 30 using probability weighted moments. Bias from STM-E is smaller in magnitude by more than $1.0 \mathrm{~m}$ for sample size $n \geq 50$ using probability weighted moments. In terms of uncertainty, STM-E always produces more realistic estimates of return value uncertainty that single location analysis. For sample sizes $n=50$ and 60, STM-E only over-predicts the inherent uncertainty of the return value by some $10-30 \%$. In conclusion, if a single location approach had been adopted for the current application, the resulting uncertainty over-estimation would have been more than $100 \%$, and the bias could have reach values up to $1.50 \mathrm{~m}$. We hope that the table provides some general guidance about the expected future performance of STM-E for locations similar to Guadeloupe archipelago, compared with single location analysis.

It is important to keep in mind that an appropriate choice of sample size $n$ for STM-E analysis is likely to be related to the size $n_{0}$ of the full sample available, and the period $T_{0}$ to which the sample corresponds. For example, in the current work, $n=20$ (and $n_{0}=124$ ) is approximately equivalent to the largest $15 \%$ of cyclones for the sample period $T_{0}=200$ year. That is, the smallest cyclone considered in the $n=20$ STM-E model has a return period of the order of 10 years. With $n=60$, we use approximately half the sample for STM-E analysis, and the smallest cyclone in the STM-E analysis has a return period of the order of 3 years. In another application where $T_{0}=50\left(\right.$ and $\left.n_{0}=32\right)$ say, it is likely that a greater proportion of the sample would need to be used to estimate the STM-E model reasonably.

The table, combined with the validation experiments reported above, confirm the principle intuited by previous works' results (Wada et al. 2018, Wada et al. 2020) that STM-E provides improved estimates of return values compared to statistical analysis of cyclone data at a single location. From an operational perspective, this means that for regions like the SW Pacific 
https://doi.org/10.5194/nhess-2021-94

Preprint. Discussion started: 29 March 2021

(C) Author(s) 2021. CC BY 4.0 License.

\begin{tabular}{|l|r|r|r|r|r|}
\hline Maximum likelihood & $n=20$ & 30 & 40 & 50 & 60 \\
\hline Bias median STM-E & -1.048 & -1.127 & -1.148 & -0.941 & -0.715 \\
\hline Bias median for single location & -1.381 & -1.333 & -1.282 & -1.339 & -1.400 \\
\hline Ratio of 95\% intervals STM-E & 0.792 & 0.617 & 0.393 & 0.216 & 0.121 \\
\hline Ratio of 95\% intervals for single location & 1.166 & 1.452 & 1.425 & 1.151 & 1.118 \\
\hline Probability weighted moments & $n=20$ & 30 & 40 & 50 & 60 \\
\hline Bias median STM-E & -1.004 & -1.014 & -0.854 & -0.441 & -0.186 \\
\hline Bias median for single location & -0.853 & -0.951 & -1.102 & -1.464 & -1.697 \\
\hline Ratio of 95\% intervals STM-E & 0.749 & 0.614 & 0.423 & 0.270 & 0.219 \\
\hline Ratio of 95\% intervals for single location & 1.165 & 1.295 & 1.421 & 1.287 & 1.247 \\
\hline
\end{tabular}

Table 1. Performance of STM-E and single-location analysis in estimation of the bias and uncertainty of the 500-year return value, relative to empirical estimates from the full synthetic cyclone data, for analysis sample sizes of 20,30, 40,50 and 60. Bias $B$ is assessed as the average difference (over the iso-depth contour and line transect analyses) between the median STM-E (or single-location) estimate and the median estimate from the full cyclone data, for that sample size. Similarly, uncertainty $U$ is assessed in terms of the average ratio of the width of the 95\% uncertainty band of the STM-E (or single-location) estimate to the width of corresponding uncertainty band from the full cyclone data, minus one. Thus, zero values of $B$ and $U$ indicate agreement between the model-based inference and that from resampling the full synthetic cyclone database.

ocean (McInnes et al. 2014) or Indian Ocean basin (Lecacheux et al. 2012) where cyclone-induced storm wave data is limited, STM-E achieves low bias and realistic levels of uncertainty, and should be preferred to the single location approach. It confirms that STM-E can be considered a valuable tool for any coastal risk practitioner.

Data availability. Sample wave data for the analysis are available on Zenodo at Krien et al. (2021).

Author contributions. Conceptualization (JR, RW, PhJ), Cyclone and wave modelling (YK, JR), Statistical methodology and modelling (RW, $\mathrm{PhJ})$, Writing (PhJ, JR, RW, YK)

Competing interests. The authors declare that they have no conflict of interest. 
https://doi.org/10.5194/nhess-2021-94

Preprint. Discussion started: 29 March 2021

(C) Author(s) 2021. CC BY 4.0 License.

355 Acknowledgements. Numerical simulations were conducted using the computational resources of the C3I (Centre Commun de Calcul Intensif) in Guadeloupe. JR acknowledges the financial funding of the Carib-Coast INTERREG project (https://www.interreg-caraibes.fr/caribcoast). MATLAB code for the analysis is provided on GitHub at Wada et al. (2021). Sample wave data for the analysis are available on Zenodo at Krien et al. (2021). 
https://doi.org/10.5194/nhess-2021-94

Preprint. Discussion started: 29 March 2021

(c) Author(s) 2021. CC BY 4.0 License.

\section{References}

Barbier, E. B.: Policy: Hurricane Katrina's lessons for the world, Nature News, 524, 285, 2015.

Bloemendaal, N., Muis, S., Haarsma, R. J., Verlaan, M., Apecechea, M. I., de Moel, H., Ward, P. J., and Aerts, J. C.: Global modeling of tropical cyclone storm surges using high-resolution forecasts, Climate Dynamics, 52, 5031-5044, 2019.

Bloemendaal, N., Haigh, I. D., de Moel, H., Muis, S., Haarsma, R. J., and Aerts, J. C.: Generation of a global synthetic tropical cyclone hazard dataset using STORM, Scientific Data, 7, 1-12, 2020.

Coles, S., Bawa, J., Trenner, L., and Dorazio, P.: An introduction to statistical modeling of extreme values, vol. 208, Springer, 2001.

Dasgupta, R., Basu, M., Kumar, P., Johnson, B. A., Mitra, B. K., Avtar, R., and Shaw, R.: A rapid indicator-based assessment of foreign resident preparedness in Japan during Typhoon Hagibis, International Journal of Disaster Risk Reduction, 51, 101849, https://doi.org/https://doi.org/10.1016/j.ijdrr.2020.101849, 2020.

Davison, A. C.: Statistical models, Cambridge University Press, Cambridge, UK, 2003.

de Zea Bermudez, P. and Kotz, S.: Parameter estimation of the generalized Pareto distribution-Part I, J. Stat. Plan. Inference, 140, 1353-1373, 2010a.

de Zea Bermudez, P. and Kotz, S.: Parameter estimation of the generalized Pareto distribution-Part II, J. Stat. Plan. Inference, 140, 1374-1388, 2010b.

Desarthe, J.: Ouragans et submersions dans les Antilles françaises (XVIIe-XXe siècle), Études caribéennes, 2015.

Emanuel, K., Ravela, S., Vivant, E., and Risi, C.: A statistical deterministic approach to hurricane risk assessment, Bulletin of the American Meteorological Society, 87, 299-314, 2006.

Furrer, R. and Naveau, P.: Probability weighted moments properties for small samples, Stat. Probab. Letters, 70, 190-195, 2007.

Hosking, J. R. M. and Wallis, J. R.: Parameter and Quantile Estimation for the Generalized Pareto Distribution, Technometrics, 29, 339-349, 1987.

Jevrejeva, S., Bricheno, L., Brown, J., Byrne, D., De Dominicis, M., Matthews, A., Rynders, S., Palanisamy, H., and Wolf, J.: Quantifying processes contributing to coastal hazards to inform coastal climate resilience assessments, demonstrated for the Caribbean Sea, Natural Hazards and Earth System Sciences, 2020.

Jonathan, P., Randell, D., Wadsworth, J., and Tawn, J.: Uncertainties in return values from extreme value analysis of peaks over threshold using the generalised Pareto distribution, Ocean Eng., 220, 107 725, 2021.

Kennedy, A. B., Westerink, J. J., Smith, J. M., Hope, M. E., Hartman, M., Taflanidis, A. A., Tanaka, S., Westerink, H., Cheung, K. F., Smith, T., et al.: Tropical cyclone inundation potential on the Hawaiian Islands of Oahu and Kauai, Ocean Modelling, 52, 54-68, 2012.

Knapp, K. R., Kruk, M. C., Levinson, D. H., Diamond, H. J., and Neumann, C. J.: The international best track archive for climate stewardship (IBTrACS) unifying tropical cyclone data, Bulletin of the American Meteorological Society, 91, 363-376, 2010.

Koussoula-Bonneton, A.: Le passage dévastateur d'un ouragan: conséquences socio-économiques. Le cas du cyclone Hugo en Guadeloupe, La Météorologie, 1994.

Krien, Y., Dudon, B., Roger, J., and Zahibo, N.: Probabilistic hurricane-induced storm surge hazard assessment in Guadeloupe, Lesser Antilles, Natural Hazards and Earth System Sciences, 15, 1711-1720, 2015.

Krien, Y., Wada, R., Rohmer, J., and Jonathan, P.: Synthetic tropical cyclone data for the Caribbean Sea, https://doi.org/10.5281/zenodo. 4627903, 2021. 
https://doi.org/10.5194/nhess-2021-94

Preprint. Discussion started: 29 March 2021

(c) Author(s) 2021. CC BY 4.0 License.

Landsea, C. W. and Franklin, J. L.: Atlantic hurricane database uncertainty and presentation of a new database format, Monthly Weather Review, 141, 3576-3592, 2013.

Lecacheux, S., Pedreros, R., Le Cozannet, G., Thiébot, J., De La Torre, Y., Bulteau, T., and Tinti, S.: A method to characterize the different extreme waves for islands exposed to various wave regimes: a case study devoted to Reunion Island., Natural Hazards \& Earth System Sciences, 12, 2012.

Lecacheux, S., Rohmer, J., Paris, F., Pedreros, R., Quetelard, H., and Bonnardot, F.: Toward the probabilistic forecasting of cyclone-induced marine flooding by overtopping at Reunion Island aided by a time-varying random-forest classification approach, Natural Hazards, 105, 227-251, 2021.

Lin, N., Emanuel, K., Oppenheimer, M., and Vanmarcke, E.: Physically based assessment of hurricane surge threat under climate change, Nature Climate Change, 2, 462-467, 2012.

405 McInnes, K. L., Walsh, K. J., Hoeke, R. K., O’Grady, J. G., Colberg, F., and Hubbert, G. D.: Quantifying storm tide risk in Fiji due to climate variability and change, Global and Planetary Change, 116, 115-129, 2014.

Merrifield, M., Becker, J., Ford, M., and Yao, Y.: Observations and estimates of wave-driven water level extremes at the Marshall Islands, Geophysical Research Letters, 41, 7245-7253, 2014.

Nadal-Caraballo, N. C., Campbell, M. O., Gonzalez, V. M., Torres, M. J., Melby, J. A., and Taflanidis, A. A.: Coastal Hazards System: A Probabilistic Coastal Hazard Analysis Framework, Journal of Coastal Research, 95, 1211-1216, 2020.

Simpson, R. H. and Saffir, H.: The hurricane disaster potential scale, Weatherwise, 27, 169, 1974.

Stephens, S. A. and Ramsay, D.: Extreme cyclone wave climate in the Southwest Pacific Ocean: Influence of the El Niño Southern Oscillation and projected climate change, Global and Planetary Change, 123, 13-26, 2014.

Vickery, P., Skerlj, P., and Twisdale, L.: Simulation of hurricane risk in the US using empirical track model, Journal of structural engineering, 126, 1222-1237, 2000.

Wada, R., Waseda, T., and Jonathan, P.: A simple spatial model for extreme tropical cyclone seas, Ocean Engineering, 169, 315-325, 2018.

Wada, R., Jonathan, P., Waseda, T., and Fan, S.: Estimating extreme waves in the Gulf of Mexico using a simple spatial extremes model, Proc. 38th Int. Conf. on Ocean, Offshore \& Arctic Engineering, Scotland, 2019.

Wada, R., Jonathan, P., and Waseda, T.: Spatial Features of Extreme Waves in Gulf of Mexico, in: ASME 2020 39th International Conference on Ocean, Offshore and Arctic Engineering, American Society of Mechanical Engineers Digital Collection, 2020.

Wada, R., Rohmer, J., Krien, Y., and Jonathan, P.: STM-E (space-time maxima and exposure) spatial extremes model for tropical cyclones, github.com/ygraigarw/STM-E, 2021.

Zahibo, N., Pelinovsky, E., Talipova, T., Rabinovich, A., Kurkin, A., and Nikolkina, I.: Statistical analysis of cyclone hazard for Guadeloupe, Lesser Antilles, Atmospheric research, 84, 13-29, 2007. 